\title{
Notch Signaling Activation Promotes Seizure Activity in Temporal Lobe Epilepsy
}

\author{
Longze Sha $\cdot$ Xiaofeng Wu Yuan Yao $\cdot$ Bo Wen • Jing Feng $\cdot$ Zhiqiang Sha • \\ Xueqin Wang • Xiaoliang Xing • Wanchen Dou • Liri Jin • Wenting Li • \\ Naili Wang • Yan Shen • Jinhui Wang • Liwen Wu • Qi Xu
}

Received: 17 June 2013 / Accepted: 19 August 2013 / Published online: 3 September 2013

(C) Springer Science+Business Media New York 2013

\begin{abstract}
Notch signaling in the nervous system is often regarded as a developmental pathway. However, recent studies have suggested that Notch is associated with neuronal discharges. Here, focusing on temporal lobe epilepsy, we found that Notch signaling was activated in the kainic acid (KA)-induced epilepsy model and in human epileptogenic tissues. Using an acute model of seizures, we showed that DAPT, an inhibitor of Notch, inhibited ictal activity. In contrast, pretreatment with exogenous Jagged1 to elevate Notch signaling before KA application had proconvulsant effects. In vivo, we demonstrated that the impacts of activated Notch signaling on seizures can in part be attributed to the regulatory role of Notch signaling on excitatory synaptic activity in CA1 pyramidal neurons. In vitro, we found that DAPT treatment impaired synaptic vesicle endocytosis in cultured hippocampal neurons. Taken together, our findings suggest a correlation
\end{abstract}

Authors Longze Sha and Xiaofeng Wu contributed equally to this work.

Electronic supplementary material The online version of this article (doi:10.1007/s12035-013-8545-0) contains supplementary material, which is available to authorized users.

L. Sha $\cdot$ X. Wu $\cdot$ Z. Sha $\cdot$ X. Wang $\cdot$ X. Xing $\cdot$ W. Li $\cdot$ N. Wang $\cdot$

Y. Shen $\cdot$ Q. Xu $(\bowtie)$

National Laboratory of Medical Molecular Biology, Institute of Basic

Medical Sciences, Chinese Academy of Medical Sciences and

Peking Union Medical College, Beijing, China 100730

e-mail: xuqi@pumc.edu.cn

Y. Yao $\cdot$ W. Dou $\cdot$ L. Jin $\cdot$ L. Wu

Department of Neurology, Peking Union Medical College Hospital, Chinese Academy of Medical Sciences and Peking Union Medical College, Beijing, China 100730

L. Wu

e-mail:wlw1946@yahoo.com.cn

B. Wen $\cdot$ J. Feng $\cdot$ J. Wang

State Key Laboratory of Brain and Cognitive Sciences, Institute of

Biophysics, Chinese Academy of Sciences, Beijing, China 100101 between aberrant Notch signaling and epileptic seizures. Notch signaling is up-regulated in response to seizure activity, and its activation further promotes neuronal excitation of CA1 pyramidal neurons in acute seizures.

Keywords DAPT $\cdot$ Notch $\cdot$ Seizures $\cdot$ Temporal lobe epilepsy

\section{Introduction}

Notch signaling regulates many developmental events of the central nervous system (CNS), including maintenance of neural stem cells (NSCs), cell fate determination, and regulation of neurite outgrowth in post-mitotic neurons [1,2]. Recent studies have implicated Notch signaling in short-term plasticity and neuronal discharges. Gaiano and colleagues reported that Notch signaling was activated by neuronal activity, as indicated by an increase in Notch1 expression and cleaved NICD1 (Notch intracellular domain 1) in cultured hippocampal neurons stimulated with NMDA (N-methyl-D-aspartate) [3]. Struhl and colleagues showed that Notch signaling in Drosophila was activated specifically by odor stimulation in brain regions responsible for olfactory processing [4]. Although these findings suggest a potential role of Notch signaling in regulating neuronal excitability, further studies are needed to better understand the role of Notch signaling in short-term plasticity and in diseases beyond those related to neuronal development.

Disruption of Notch signaling has been described in an epileptic mind bomb zebrafish which are characterized by a severe neurogenic phenotype and spontaneous seizures. These defects have been interpreted as a consequence of abnormal Notch signaling and developmental disability of the nervous system [5-7]. However, given the possible role of Notch signaling in regulating neuronal discharges in mature neurons, it remains unclear whether Notch signaling may participate in seizure generation. In the present study, we used mouse model of acquired 
epilepsy to study the spatial and temporal activation pattern of Notch signaling at different stages of temporal lobe epileptogenesis. Using an acute model of seizures, we provide evidences that Notch is activated in response to seizure activity and that its activation promotes seizures, partly by elevating excitatory synaptic transmission of CA1 pyramidal neurons. Furthermore, we show that DAPT, which inhibits Notch signaling through $\gamma$-secretase, can suppress acute seizures, as well as inhibit synaptic vesicle generation in cultured hippocampal neurons.

\section{Results and Discussion}

Activation of Notch Signaling in KA-Induced Mouse Model of Epilepsy

To examine whether seizure activity affects Notch activation, we used the well-established kainic acid (KA) mouse model of temporal lobe epilepsy $[8,9]$. After unilateral intrahippocampal injection of $200 \mathrm{ng} \mathrm{KA}$, mice experienced at least $2 \mathrm{~h}$ of convulsive status epilepticus (SE), and recurrent seizure activity developed within 1 week. We measured Notch1, NICD1, and Jagged1 by western blots of hippocampal homogenates at various time points in the first week after inducing SE by KA injection; vehicle-injected hippocampus served as control group. In the acute phase, a significant increase in Notch1 and Jagged1 was seen within $3 \mathrm{~h}$ of the onset of KA-induced seizures (Fig. 1A,B,C,D). The expression of Notch1 peaked at $24 \mathrm{~h}$ after KA-induced seizures and was still up-regulated during the latent phase, as examined on day 5 post-SE. KA treatment led to an increase in NICD1 levels at $3 \mathrm{~h}$ and at $24 \mathrm{~h}$, and the NICD1 peaked on day 5 post-SE (Fig. 1A, B). Furthermore, Notch signaling in the hippocampus contralateral to the injection site was slightly increased during acute and latent period but returned to basal levels at 5 weeks post-SE (Online Resource 1).

\section{Up-regulation of Notch Signaling in the Dispersed Granule Cells}

Next, we investigated whether the spatial pattern of Notch signaling activation was altered in the sclerotic hippocampi from mice with chronic spontaneous seizures. Widespread distribution of NICD1 immunohistochemical staining was observed in the molecular layer of dentate gyrus which contains the dendrites of granule cells (Fig. 2A). In the fifth week after KA-induced seizures, NICD1 was more abundant in the hilus, CA1, and CA3 subfields, suggesting that NICD1 was up-regulated in neurites throughout the hippocampus (Fig. 2D). The longlasting up-regulation of Notch signaling in the chronic stage of epilepsy may result in a disruption of dendritic arborization and increased dendritic spines in neurons, as reported previously [10]. Furthermore, the seizure frequency at 12 weeks after KA- induced SE was higher than at 5 weeks post-SE $(119 \pm 20$ and 52 \pm 7 per day, respectively; Fig. $2 \mathrm{H}$ ), and this was accompanied by a higher NICD1 protein levels (Fig. 2H).

The expression of Jagged1 and Notch1 was prominent in pyramidal neurons of control hippocampus while was low expressed in the granule cells of the dentate gyrus (Fig. 2B,C). In the fifth week after KA injection, upregulation of Notch1 and Jagged1 was observed in ectopic granule cells (Fig. 2E,F), as determined by the fluorescence signals of Jagged1 and Notch1 in Tuj1-positive granule cells of the sclerotic hippocampus and control hippocampus (Fig. 2G).

Up-regulated Expression of NICD1 in Human Epileptogenic Tissues

Furthermore, we examined the protein levels of NICD1 in the sclerotic hippocampus from mesial temporal lobe epilepsy (MTLE) patients. The NICD1 levels in the hippocampal sclerosis (HS) group were increased about $2.8 \pm 0.4$-fold compared with autopsy controls, and 1.6-fold compared with the nonHS groups (Fig. 3A,B). Considering the effect of the postmortem interval (PI) on protein stability, we tested the influence of time from death to snap freezing of mouse brain tissue and found a limit change in NICD1 protein levels under the condition of a post-mortem time of $8 \mathrm{~h}$ (Online Resource 2).

Autopsy controls showed that NICD1 staining was almost absent from the neurites of granule cells (Fig. 3C). The same was true of five of the non-HS cases (Fig. 3D). In the resected hippocampi of patients with HS, strong NICD1 immunoreactivity was present in the molecular layer and the hilus of the dentate gyrus which mainly contains the dendrites and the mossy fiber of granule cells, respectively (Fig. 3E). The inset of Fig. 3E showed strong NICD1 immunoreactivity in neurites of granule cells. The study of Notch signaling in the sclerotic hippocampi from TLE patients verified a similar activation pattern which was observed in the mouse model of chronic epilepsy.

Application of Exogenous Jagged1 Promotes Acute Seizures

To determine whether the activation of Notch signaling induced by acute epilepsy was involved in seizure generation, we applied pharmacological interference on an acute model of epilepsy in which seizures were induced by intrahippocampal application of $7 \mathrm{ng}$ KA. This dose induced electroencephalography (EEG) epileptiform activity in $100 \%$ of mice without any mortality or apparent neuronal loss [11, 12].

A schematic illustration of the experimental protocol is presented in Fig. 4A. Ictal activity was confirmed by both video-EEG recordings and calculated by summing the duration of each ictal episode, which included behavioral arrest and generation of a clonic seizure (Online Resource 3). Recombinant Jagged1 protein was intrahippocampally injected 30 min prior to KA injection to evoke Notch signaling (Fig. 4B). 

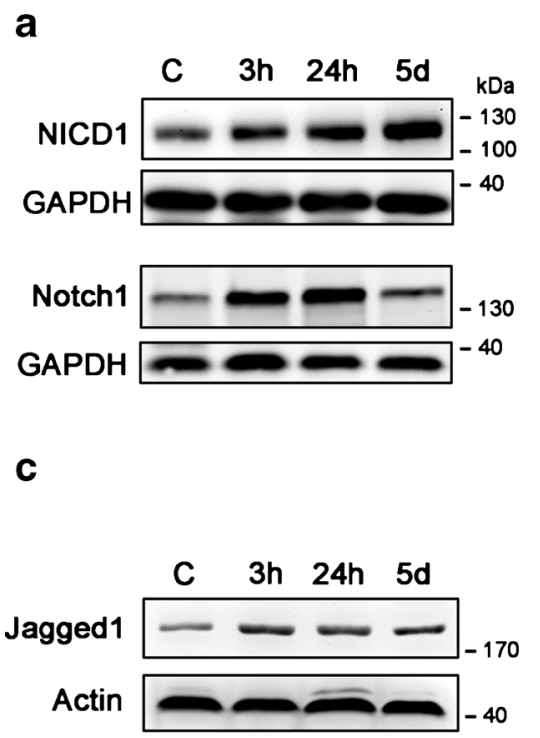

Fig. 1 Notch signaling was activated by KA-induced seizures. A Western blotting showed Notch1 expression and NICD1 levels in the injected hippocampus at different time interval ( $3 \mathrm{~h}, 24 \mathrm{~h}, 5$ days) within the first week of KA-induced seizures (which lasted $4.2 \pm 1.4 \mathrm{~h}$ ). Vehicle-injected hippocampus served as control group. B Quantitative analyses demonstrated that the expression levels of Notch1 and NICD1 were up-regulated

Jagged1 injection significantly increased the ictal activity $5.6 \pm$ 1.4-fold and the number of seizures $3.0 \pm 0.7$-fold compared with vehicle-injected mice (Fig. 4C). However, activation of Notch signaling did not influence the time to onset of the first seizure (Fig. 4C).

\section{Anticonvulsant Effects of DAPT Application Prior to KA-Induced Seizures}

The results above predict that the pharmacological blockade of Notch signaling should have anticonvulsant effects. We therefore interfered with the activation of Notch signaling by injecting DAPT, a $\gamma$-secretase inhibitor, to suppress KA-induced Notch receptor cleavage (Fig. 4D). Compared with the vehicle group, DAPT injection reduced the duration of KA-induced seizures by $72 \%$ and the number of seizures by $56 \%$ (Fig. 4E). These observations suggest that Notch signaling activation by acute seizures in turn facilitates further neuronal excitability. Conversely, as observed in Jagged1 injected group, DAPT treatment also has no effect on the onset of seizures (Fig. 5E), suggesting that activation of Notch signaling is not related to the mechanism by which seizures are triggered.

Modulation of Notch Signaling Altered the Excitability of CA1 Pyramidal Neurons

Since the distribution of uncleaved Notch1 receptors was largely restricted to pyramidal neurons in the normal b
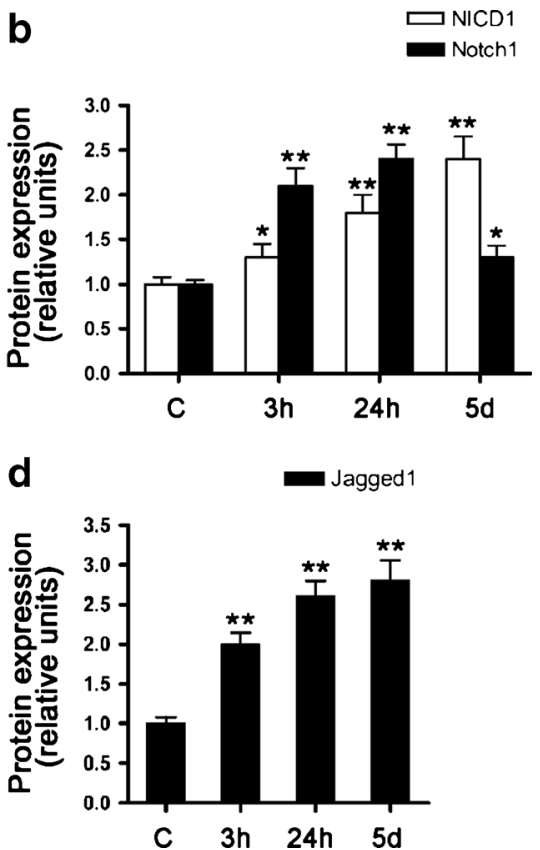

at $3 \mathrm{~h}$ after the onset of KA-induced seizures. C, D The expression of Jagged1 was increased at $3 \mathrm{~h}$ after KA-induced seizures (2.0 \pm 0.1 -fold), and it peaked ( $2.8 \pm 0.2$-fold) on the fifth day post-SE. Error bars are mean \pm SE. Single asterisk $(*) p<0.05$, double asterisk $\left({ }^{* *}\right) p<0.01$ by one-way ANOVA ( $n=5$ or 6 for each time point)

hippocampus (Fig. 2C), the increase in NICD1 induced by acute seizures may be caused mainly by the cleavage of Notch receptors in pyramidal neurons. More importantly, a previous study reported that mice with deleted Notch1 gene in CA1 pyramidal neurons exhibited reduced long-term potentiation [3]. We therefore examined whether the activation of Notch signaling in acute seizures could influence the synaptic activity of CA1 pyramidal neurons. We recorded spontaneous excitatory post-synaptic currents (sEPSC) of CA1 pyramidal neurons from KA-induced mouse model of seizures under conditions of vehicle pretreatment and Jagged1 pretreatment as described in the drug testing experiment. Figure 5A illustrates that Jagged 1 pretreatment appears SEPSC amplitude and frequency in hippocampal CA1 pyramidal neurons. The statistical analyses about cumulative probability versus interEPSC intervals (Fig. 5B) and versus sEPSC amplitudes (Fig. 5C) indicate that Jagged1 pretreatment decreases interEPSC intervals and increases sEPSC amplitudes compared with vehicle-pretreated CA1 pyramidal neurons. By contrast, DAPT pretreatment decreased sEPSC amplitudes and increased inter-EPSC intervals (Fig. 5D,E,F).

Moreover, we found that neither Jagged1 nor DAPT treatment affects the action potential thresholds and the inputoutput curves of CA1 pyramidal neurons (Online Resource 4). These results suggest that the impact of Notch signaling activation on the functions of CA1 pyramidal neurons is mainly targeted to the signal transmission at glutamatergic synapses. Overall, the proconvulsant effect of activated 


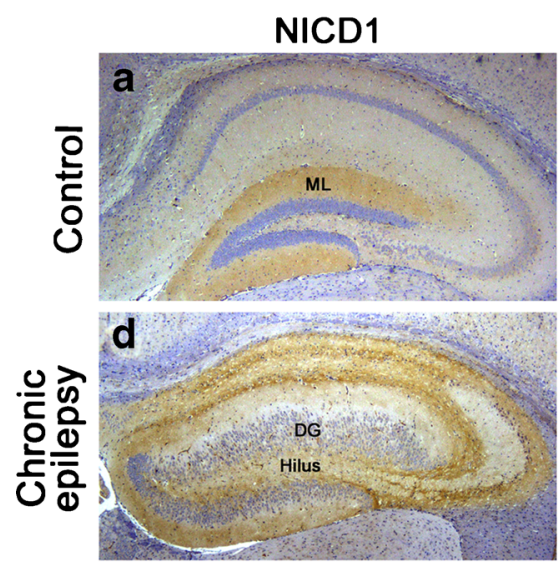

9

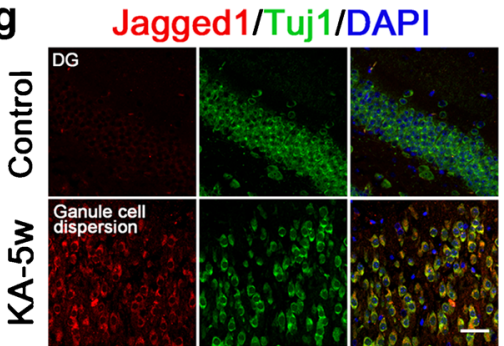

Notch1/Tuj1/DAPI

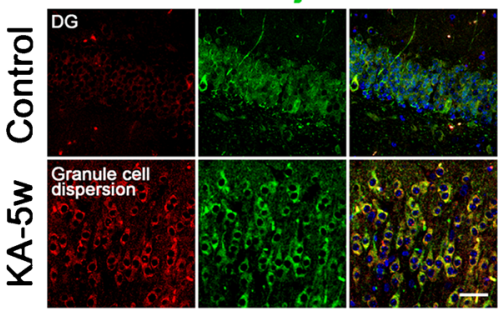

Fig. 2 Distribution of NICD1, Jagged1, and Notch1 in the sclerotic hippocampus of mice with chronic epilepsy. A In vehicle-treated control mice $(n=5)$, NICD1 immunoreactivity was detectable in the molecular layer of the dentate gyrus. D The hippocampus with long-term epilepsy showed strong NICD1 immunoreactivity throughout the hippocampal subfields ( 5 weeks after KA-induced seizures, $n=4$ ). B, C Jagged 1 and Notch1 were present in the pyramidal neurons and expressed at low levels in the granule cells of the dentate gyrus. E, F During the chronic phase, increased expression of Jagged1 and Notch1 was observed in the dispersed granule cells of KA-injected hippocampus. G Increased

Notch signaling on acute seizures can be partly attributed to a regulatory role of Notch signaling in the synaptic transmission of CA1 pyramidal neurons.

DAPT Treatment Disrupted Synaptic Vesicle Endocytosis in Cultured Hippocampal Neurons

It has been reported that Notch1 coimmunoprecipitates with Dynamin from protein lysates generated from neuronal cultures, and NICD1 protein levels is regulated by activity in vitro [3]. Dynamin is a neuronal phosphoprotein and a GTPase enzyme which is involved in clathrin-mediated endocytosis in neural cells [13], and endocytosis is a critical step to
Jagged1
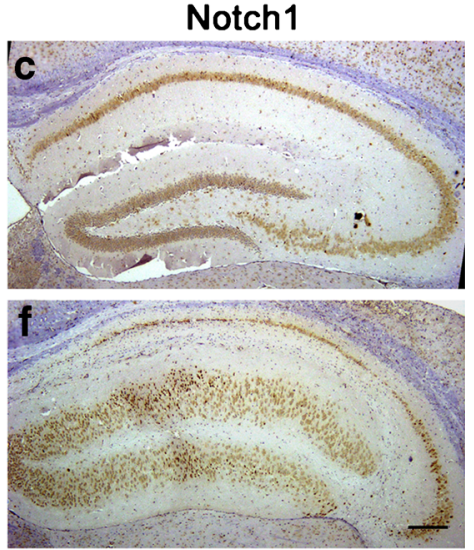

h

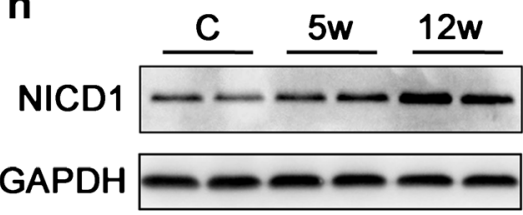

NICD1

Seizure frequency

C $5 w$

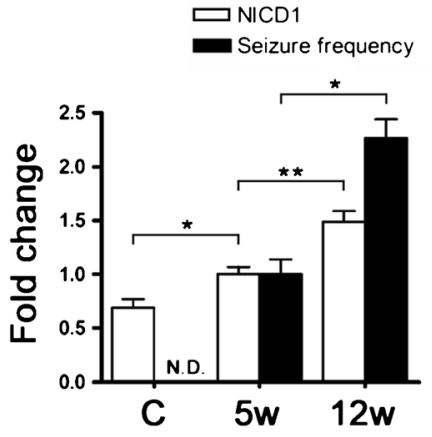

C $5 w$

expression of Jagged1 and Notch1 (red) in Tuj-1 positive neurons (green) of epileptic hippocampus compared with control hippocampus. Data were the average fluorescence intensity of 50 granule cells. H Seizure frequency of mice at 12 weeks $(n=4)$ post-SE was increased by $2.2 \pm 0.2$-fold compared with mice at 5 weeks $(n=4)$. This was accompanied with increased NICD1 expression (1.5 \pm 0.1 -fold). $M L$ molecular layer, $D G$ dentate gyrus. Scale bar in $\mathbf{A}-\mathbf{F}$ : $100 \mu \mathrm{m}$. Error bars are mean \pm SE. N.D. not detectable. Single asterisk (*) $p<0.05$, double asterisk $(* *) p<0.01$, triple asterisk $(* * *) p<0.001$ by one-way ANOVA or Student's $t$ test

generate new synaptic vesicles for neurotransmitter loading [14]. Having shown that Notch signaling is associated with synaptic activity of CA1 pyramidal neurons, we sought to confirm that disruption of Notch signaling could impair synaptic vesicle formation. FM4-64 is a membrane-selective fluorescent dye that has been widely used to observe synaptic vesicle recycling $[15,16]$. We used the dye to monitor synaptic endocytosis in conditions of KA stimulation with or without DAPT. Primary hippocampal neurons at 10 days in vitro (DIV) were treated with DAPT for $1 \mathrm{~h}$, and stimulated with KA for another $2 \mathrm{~h}$ to induce synaptic activity. For FM4-64 loading, the dye was applied to the cultures for 30 or $120 \mathrm{~s}$. After washing, the clusters of vesicles can be observed as 
a

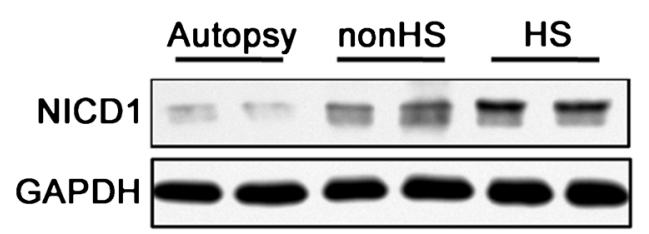

b

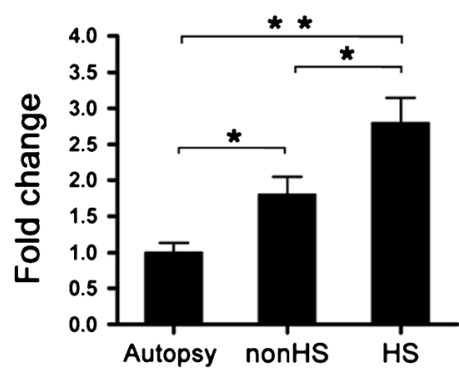

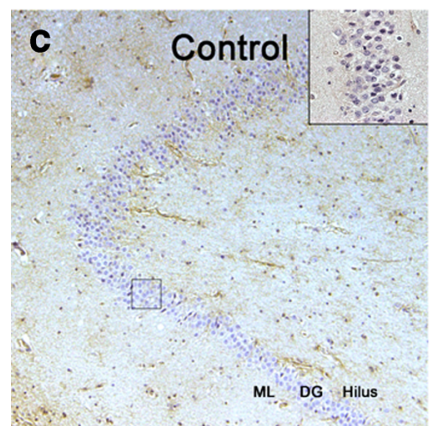

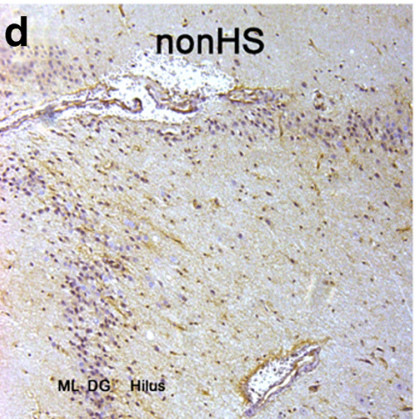

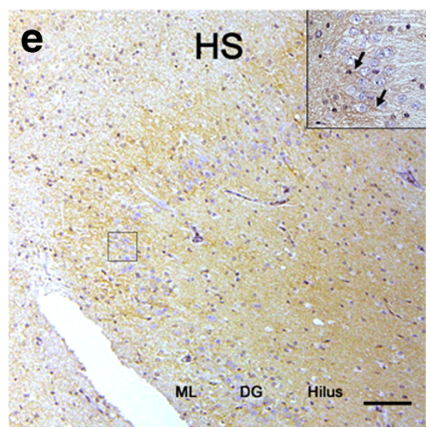

Fig. 3 Activated Notch signaling in human epileptogenic tissues. Western blot analysis of NICD1 in hippocampus: A representative immunoblots and $\mathbf{B}$ densitometric analysis of total homogenates from autopsy control hippocampi $(n=5)$, non-sclerotic controls (non-HS, $n=5)$, and sclerotic hippocampi from MTLE patients (HS, $n=7$ ). The NICD1 levels in patients with HS were significantly higher than that in autopsy controls (2.8 \pm 0.4 -fold) and also higher than non-HS group (1.6-fold). Immunohistochemical study of autopsy controls $(n=7)$, non-HS $(n=5)$, and resected hippocampi of patients with HS $(n=11)$ showed significant upregulation of NICD1 immunoreactivity in the molecular layer and the hilar region of the dentate gyrus of the sclerotic hippocampus $(\mathbf{C}, \mathbf{D}, \mathbf{E})$. Arrowhead in the inset of $\mathbf{E}$ indicated strong NICD1 immunoreactivity that was present around the somata and neurites of neurons within the granule cell layer of sclerotic hippocampus. $P I$ post-mortem interval, $M L$ molecular layer, $D G$ dentate gyrus. Scale bar in $\mathbf{C}-\mathbf{E}: 100 \mu \mathrm{m}$. Single asterisk (*) $P<0.05$, double asterisk (**) $P<0.01$ by one-way ANOVA distinct fluorescent spots along the neurites (arrows in Fig. 6A). In unstimulated cultures (Fig. 6A, images $b 1, b 4$ ), number of fluorescent spots were found to be much lower than in KA-stimulated group (Fig. 6A, images $b 2, b 3, b 5, b 6$ ). DAPT treatment significantly decreased FM4-64 signal along the neurites compared with KA treatment alone, which represented increased endocytosis process (Fig. 6A, images $b 2, b 3, b 5, b 6)$. Statistic analysis of fluorescent intensity confirmed the results (Fig. 6B). Qualification of the number of puncta along neurites also demonstrated a decreased FM4-64 uptake in the cultures that have undergone DAPT treatment (Fig. 6C,D). These results indicate that DAPT treatment could impair KA-stimulated synaptic vesicle generation in cultured hippocampal neurons.

\section{Discussion}

\section{Notch1 Is Activated in Response to Seizure Activity}

In addition to cell fate determination, recent studies have increasingly highlighted the importance of Notch signaling in the regulation of synaptic plasticity $[3,10,17,18]$. In the present study, we investigated Notch signaling in mature neurons of the hippocampus undergoing epileptogenesis and found that Notch activity was persistently elevated after induction of status epilepticus. We showed that KA-induced acute and chronic seizures activated Notch signaling. On day 5 after KA-induced seizures, the expression of Jagged1 and NICD1 reached maximum levels. The mechanism by which Notch activation reached a peak on day 5 is unclear, but this event occurs in the latent period making it potentially critical for the establishment of subsequent recurrent seizures. In the chronic stage of epileptogenesis, Notch signaling was still up-regulated in neurites throughout the hilus and CA1 subfields. We speculate that sustained Notch activation in disorganized synaptic networks may result in a long-lasting disruption of dendritic arborization of neurons and increased dendritic spines in mature neurons, as reported previously [10]. Besides, we found that the activation of Notch signaling accompanied with an increase in seizure frequency. Thus, both the temporal and spatial patterns of Notch activation suggest that aberrant Notch signaling may be involved in the formation of abnormal neural circuits in temporal lobe epileptogenesis.

Notch Signaling Activation Contributes to Perpetuating Acute Seizures

It is well known that Notch signaling plays important roles in the regulation of neuronal migration and synaptic morphology 
a

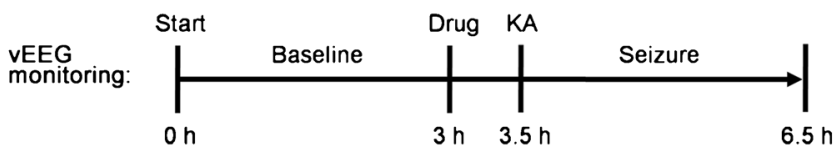

b

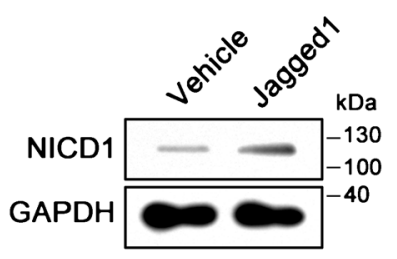

d

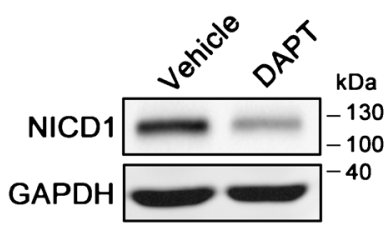

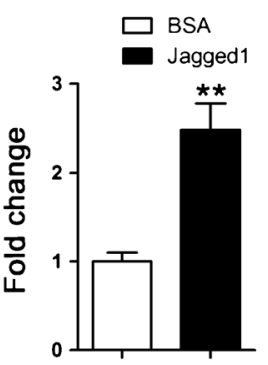

C
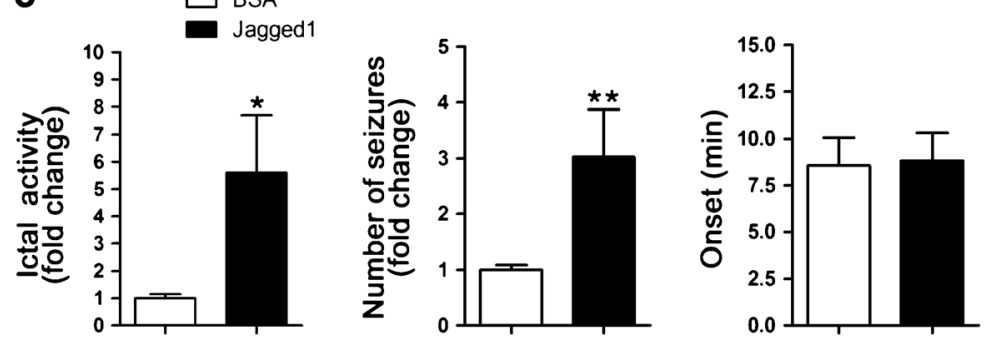

e
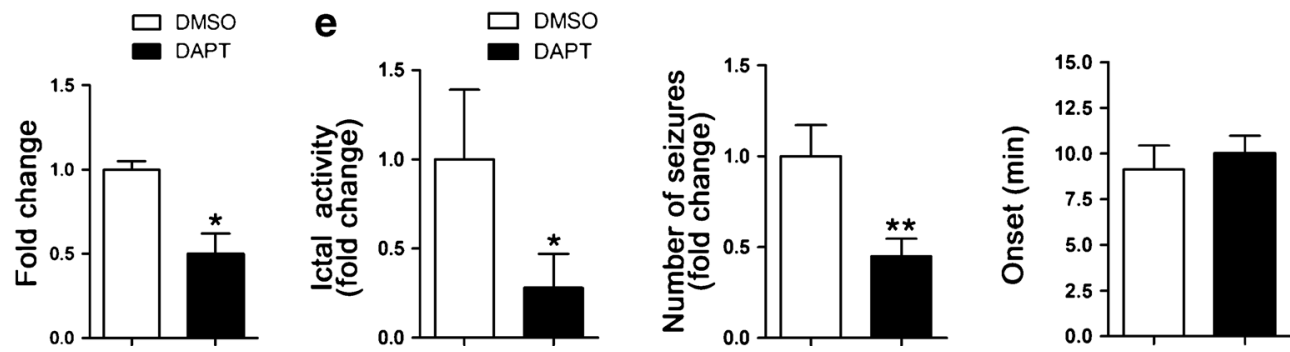

Fig. 4 The effects of exogenous Jagged 1 or DAPT on acute seizures. A Experimental protocol: the first EEG recording period ( $0-3 \mathrm{~h})$ was used to assess the baseline of mouse EEG activity before injection. Drug was injected $0.5 \mathrm{~h}$ before KA. EEG activity was then recorded continuously after KA injection (3.5-6.5 h), and EEG data were analyzed to evaluate the effects of each drug on KA-induced acute seizures. All mice were recovered from KA-induced acute seizures within $3 \mathrm{~h}$. A dose of $10 \mu \mathrm{g}$ Jagged1 was injected into the hippocampus $0.5 \mathrm{~h}$ prior to KA-induced seizures. B The NICD1 levels in the Jagged1-pretreated group were increased in about 2.5 -fold at $3 \mathrm{~h}$ post-KA injection compared with the

[2]. However, in contrast to these time-dependent changes, we observed a Jagged1 induced timely enhancement of acute seizures. This indicates that acute activation of Notch signaling may be associated with short-term synaptic plasticity. The relationship between Notch signaling and neuronal excitability has been proposed before; Gaiano and colleagues reported that NMDA treatment could activate Notch signaling in cultured hippocampal neurons, and Jagged1 and Notch1 localized at the synaptic site of mature neurons [3]. Hippocampal slices from Notch-antisense transgenic mice or in mice with a conditional, post-natal deletion of Notch1 showed decreased long-term potentiation [3, 18]. It is possible that Notch signaling may serve as a positive feedback factor for synaptic activity. In this study, we test the hypothesis by pharmaceutical interventions of Notch signaling in mouse model of acute seizures. Our finding that Jaggedl treatment promoted seizures indicates that activation of Notch signaling increases the excitability of hippocampal neurons during acute epilepsy. Furthermore, exogenous Jagged1 increased excitatory synaptic activity of CA1 pyramidal neurons without affecting intrinsic excitability suggesting that activation of Notch signaling promotes excitatory synaptic transmission.

vehicle group $(n=3)$. C Significant increases in ictal activity (measured by summing up the duration of every ictal episode) and total number of seizures were observed in Jagged1 pretreated mice $(n=8)$ compared with vehicle group $(n=11)$. D DAPT treatment reduced NICD1 levels in the hippocampus $(0.5 \pm 0.1$-fold, $n=3)$. E DAPT pretreated mice $(n=8)$ exhibited a significant decrease in ictal activity and total number of seizures but had no difference in the onset time compared with the vehicle group $(n=9)$. Error bars are mean \pm SE. Single asterisk $\left(^{*}\right) p<0.05$, double asterisk (**) $P<0.01$ by Student's $t$ test

Previous studies reported that Notch1 interact with Dynamin [3], which is critical for clathrin-mediated endocytosis in neural cells [19]. We therefore examined the effect of DAPT on synaptic vesicle generation of hippocampal neurons during KA stimulation. Using FM4-64 assay, our in vitro data show that neuronal endocytosis stimulated by KA can be blocked by DAPT, which could be responsible for its anticonvulsant effects. Taken together, the present results indicate that activation of Notch signaling during acute seizures increases synaptic activity of CA1 pyramidal neurons and predict a mechanism by which Notch signaling facilitates the release of neurotransmitter through its impact on synaptic vesicles generation, thus result in neuronal hyperexcitability. It is noteworthy that DAPT inhibits Notch signaling in an indirect manner; therefore, the anticonvulsant effects of DAPT may come from a combined suppression on several substrates of gamma secretase (e.g., EphA4) [20, 21]. Further studies are needed to analyze the extent to which inhibition of Notch signaling suppresses seizures and confirm the involvement of Notch signaling in vesicle recycling. 
a
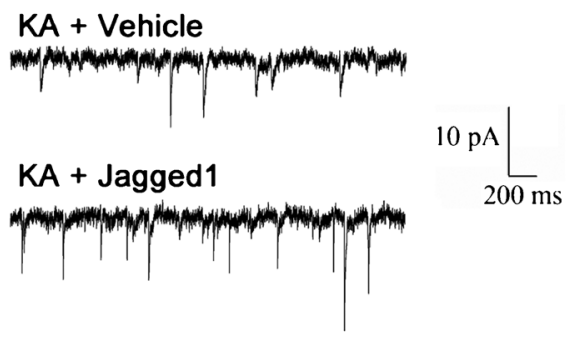

d

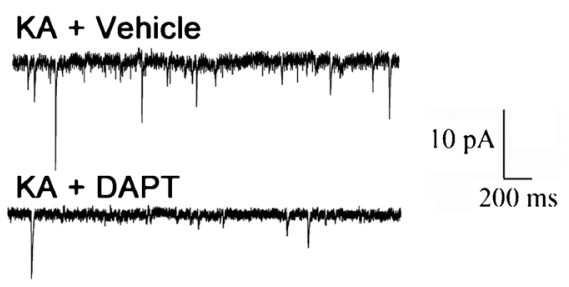

b

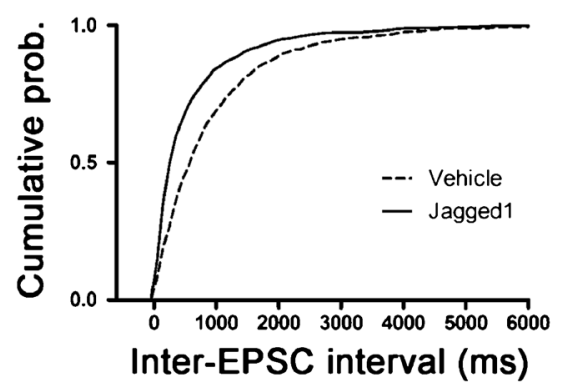

e

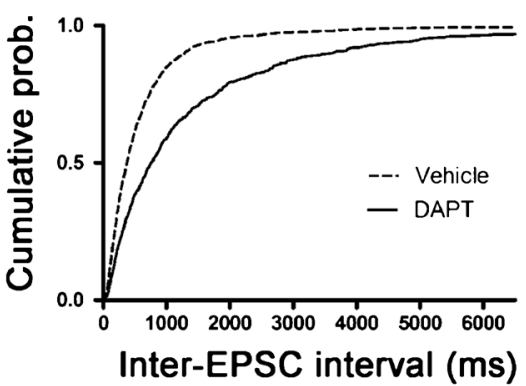

C

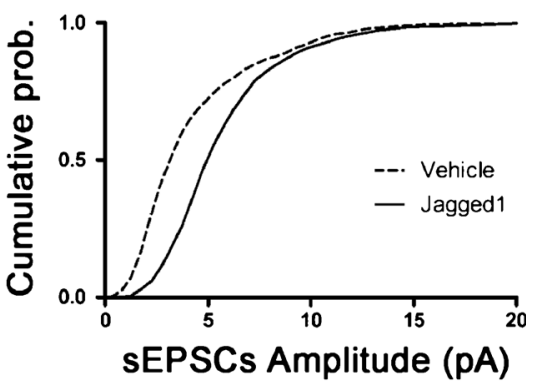

f

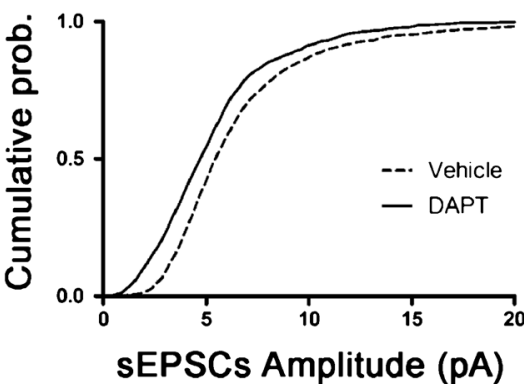

Fig. 5 Jagged 1 or DAPT treatment altered the excitability of CA1 pyramidal neurons. A sEPSC recordings from representative CA1 pyramidal neurons from vehicle and Jagged 1 pretreated mouse model of acute seizures. Cumulative probability histograms of isolated events from Jagged 1 pretreated KA mice $(n=6$ cells) demonstrated a decrease in the inter-EPSC interval $(\mathbf{B})$ and an increase in the amplitude $(\mathbf{C})$ compared with vehicle-pretreated KA mice ( $n=5$ cells, $P<10^{-4}$ by Kolmogoroff-
Smirnoff test). D sEPSC from representative CA1 pyramidal neurons from vehicle and DAPT pretreated mouse model of acute seizures. Cumulative probability histograms of isolated events from DAPT pretreated KA mice ( $n=6$ cells) showed increased inter-EPSC interval (E) and decreased amplitude (F) compared with vehicle-pretreated KA mice ( $n=6$ cells, $P<10^{-4}$ by Kolmogoroff-Smirnoff test)

coupled with an enhanced differentiation into astrocytes [30, 31]. Because of two distinct cell types, there is no contradiction between the up-regulation and down-regulation of Notch signaling in mature neurons and NSCs, respectively. Importantly, it is generally considered that recurrent seizures are responsible for the impairment of neurogenesis, and the latter leads to further establishment of the epileptogenic hippocampal circuitry and neuronal hyperexcitability [32]. Thus, both the two kinds of aberrant Notch signaling may contribute to the pathogenesis of epilepsy and schizophrenia.

In summary, our studies unravel a novel role of Notch signaling as a contributing factor for the development of acute seizures in MTLE and highlight that modulation of Notch signaling as a molecular mechanism to adjust synaptic dynamics. However, Notch signaling activation is a long-lasting process; the mechanism that underling the proconvulsant effects of Notch signaling in acute epilepsy is not suitable for chronic epilepsy due to a shifted spatial distribution of upregulated Notch molecules. Moreover, neuronal death in CA1 pyramidal layer makes this type of neurons absent from the generation of chronic seizures; thereby, the roles of Notch signaling in dentate gyrus during chronic seizures needs to replenish. 

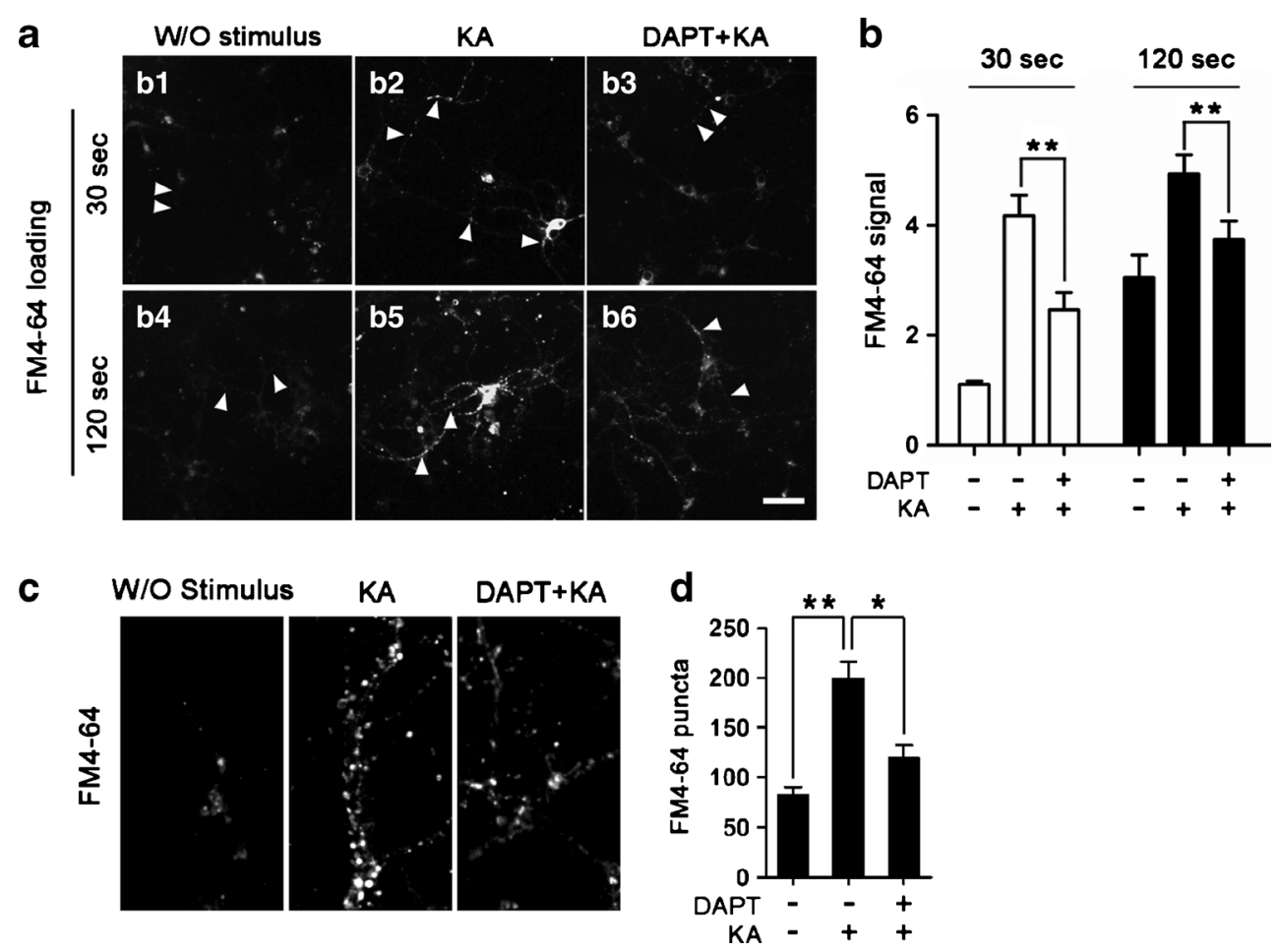

Fig. 6 DAPT treatment disrupted KA-induced synaptic endocytosis in vitro. A Representative images of FM4-64 loading in neuronal cultures under conditions of vehicle (W/O stimulus), KA stimulus, or DAPT plus KA stimulus. All fluorescent images were acquired under same exposure parameters. Neuronal cultures without KA stimulation appeared less number of fluorescent spots along the neurites (arrowheads in b1). KA stimulation resulted in an increased FM4-64 loading as shown by more spots along the neurites (arrowheads in b2), whereas DAPT treatment

before (60 min) significantly reduced FM4-64 signal ( $b 3$ ). Similar results were observed when a longer FM4-64 loading time was applied (b4-b6). B Difference in the average fluorescent signal of each group. C, D Images of FM4-64 puncta along neurites and statistical analysis of the pictures used for the measurement of fluorescence intensity (120-s loading). Error bars are mean \pm SE. Single asterisk $(*) P<0.05$, double asterisk $\left.{ }^{* *}\right) p<0.01$ by one-way ANOVA

\section{Methods}

\section{Ethics Statement}

All patients gave written informed consent prior to participation in this study; all procedures applied to this study were approved by the Ethics Committee of Chinese Academy of Medical Sciences, Peking Union Medical College, and the ethics committee of the Peking Union Medical College Hospital (PUMCH). All animal work was approved by the Experimental Animal Center of Peking Union Medical College and in accordance with the institutional guidelines of the Beijing Administration Office of Laboratory Animals.

Electrodes Implantation, Mouse Model of Seizures, and Video-EEG Analysis

We used adult male C57BL/6 mice (Vital River Animal Technology Co., Ltd., Beijing, China) weighing 18-25 g (810 weeks old) for epilepsy model, drugs testing, and EEG

monitoring. Bipolar depth electrodes $(60 \mu \mathrm{m}$ OD) were implanted into the right dorsal hippocampus [anteroposterior $(\mathrm{AP})=-2.0 \mathrm{~mm}$, mediolateral $(\mathrm{ML})=-1.8 \mathrm{~mm}$, dorsoventral $(\mathrm{DV})=-2.3 \mathrm{~mm}]$. A 23 -gauge cannula was unilaterally positioned on the top of dura mater and glued to the depth electrode for the intrahippocampal infusion of drugs (RWD, China, \#62003). We also inserted one screw serving as the ground electrode into the skull through a drilled hole without piercing the dura ( $\mathrm{AP}=-4.5 \mathrm{~mm}, \mathrm{ML}=1.5 \mathrm{~mm})$. We fixed the cannula, electrodes, and screws with bone cement containing antibiotics (DePuy, Leeds, UK). Mice were allowed to have a post-operative recovery period of 7 days before staring the drug study [12]. Time-locked digital video was recorded with a video camera when mouse EEG activities were monitored [33]. We induced acute seizures by intrahippocampal injection of $7 \mathrm{ng}$ kainic acid (KA, Sigma-Aldrich, Shanghai, China) in $0.5 \mu \mathrm{l}$ PBS[34]. The time at which mice exhibited behavioral arrest, stereotypies, limb clonus, or general tonic-clonic seizures accompanied with epileptiform 
discharges was noted and then used to analyze the corresponding seizure parameters $[11,12]$. Mice used to establish chronic model of seizures were injected with $200 \mathrm{ng} \mathrm{KA}$ in $50 \mathrm{nl}$ saline into the right dorsal hippocampus as described before $[8,9]$.

\section{Acute Model of Seizures and vEEG Analysis of Seizures}

Eight to 11 C57BL/6 mice were used in each group. Before application of any drugs, we recorded video-EEG (vEEG) in free moving mice for $30 \mathrm{~min}$ to establish a baseline EEG activity. Thirty minutes before seizure induction, we injected DAPT (D5942, Sigma, St. Louis, MO, USA) or recombinant Jagged1 (599-JG-100, RD, Littleton, CO, USA) unilaterally into the hippocampus $(0.5 \mu \mathrm{l} / 2 \mathrm{~min})$ with a needle protruding $2.3 \mathrm{~mm}$ from the guide cannula and connected to a $1-\mu 1$ Hamilton microsyringe. DAPT was dissolved in $0.5 \mu \mathrm{PBS}$ (pH 7.4) for a concentration of $50 \mu \mathrm{M}$, and PBS containing $0.5 \%$ DMSO was used as the vehicle. For Jagged1 group, $10 \mu \mathrm{g}$ recombinant protein was dissolved in $1 \mu \mathrm{lBSS}$ ( $\mathrm{pH}$ 7.4), and PBS containing $10 \mu \mathrm{g}$ BSA was used as the vehicle. At the end of the injection, the needle remained in place for at least $1 \mathrm{~min}$ to avoid reflux.

After KA injection, seizures occurred within $15 \mathrm{~min}$. Epileptiform activity was characterized by isolated spikes, polyspikes, and paroxysmal discharges $(1-5 \mathrm{~Hz}$, high voltage rhythmic activity or 10-14 Hz, low voltage rhythmic activity) that lasted at least $10 \mathrm{~s}$. Video information was analyzed as needed to confirm behavioral correlating with seizures and to rule out electromyographic artifact. For drug testing in acute model of seizures, each mouse was recorded for $3 \mathrm{~h}$. All videoEEG data were analyzed by two independent trained researchers.

The onset time of seizure (abbreviated as onset in figures) is defined by time elapsed from KA injection to the occurrence of the first EEG seizure. Ictal activity was measured by summing up the duration of every ictal episode during the EEG recording period. Number of seizure refers to the total number of seizures after KA application. Time in seizures was measured by summing up the duration from the first to the last seizure event. Time of each ictal episode was measured by averaging each seizure duration.

\section{Model of Chronic Spontaneous Seizures}

Two months after KA-induced status epilepticus (SE), mice were implanted with the electrodes injected into the hippocampus to detect EEG. Three quarters of mice exhibited spontaneous EEG seizure, and the chronic epileptic group was then disconnected from the EEG recording setup and prepared for the following immunohistochemical studies.

To evaluate the frequency of spontaneous seizures, we EEG-monitored the mice every day from 9:00 to 18:00, for four consecutive days, to calculate the total number of seizures per day (seizure frequency).
Tissues from Patients with TLE and Autopsy

Surgically resected sclerotic hippocampi used in this study were obtained from eight patients with intractable TLE, who underwent surgical treatment at PUMCH. All patients $(n=11)$ were diagnosed with HS according to high-resonance magnetic resonance imaging measurements, interictal FDG-PET, EEG recording, and histopathologic examination. In the other five patients, focal lesions such as gliomas, cortical dysplasia, or oligodendroglioma was observed but did not involve the hippocampus, and histological examination revealed no significant hippocampal neuronal loss in these patients (non-HS). The non-HS subjects were used as a control group for comparison with the HS cases. Control hippocampal tissues were obtained from autopsy of six patients without history of epilepsy or other neurological diseases. All autopsies were performed within $8 \mathrm{~h}$ after death.

Surgically resected tissues were immediately stored below $10{ }^{\circ} \mathrm{C}$ and treated with $10 \%$ buffered formalin within $1 \mathrm{~h}$ after surgery, fixed no later than $48 \mathrm{~h}$, and then embedded in paraffin. Paraffin-embedded tissues were sectioned at $4 \mu \mathrm{m}$ for immunohistochemistry studies. Parts of the tissue samples from mTLE patients and autopsy were stored in liquid nitrogen until further Western blot analysis was performed.

Evaluation of Loss NICD1 Protein Level in the Mouse Brain Post-Mortem

To evaluate the possible loss in NICD1 protein level postmortem, we performed an experiment in which mice brains were exposed for $8 \mathrm{~h}$ at $10^{\circ} \mathrm{C}$. Mice brains were kept within their sculls 0 and $8 \mathrm{~h}$ ( $n=6$ for each group) as described for the human brain samples, and then their hippocampus was removed for subsequent western blot analysis. To avoid potential degradation of housekeepping proteins, we also used coomassie staining as loading control in Western blot analysis.

\section{Western Blot Analysis}

Mice were sacrificed by decapitation under deep anesthesia with pentobarbital. The hippocampus was rapidly dissected and frozen. For immunoblot analysis, the hippocampus dissected from humans or mice ( $n=5$ or 6 ) was homogenized in RIPA lysis buffer. The following primary antibodies were used: anti-Notch1 (1:500, \#sc-32745, \#SC-23299, Santa Cruz Biotechnology, Santa Cruz, CA, USA), anti-Jagged1 (1:500, \#SC-8303, Santa Cruz Biotechnology, Santa Cruz), anti-Notch1 intracellular domain (1:1,000, \#1935-1, Epitomics, Burlingame, CA, USA), anti-GAPDH $(1: 1,000$, \#2118, Cell Signaling, Danvers, MA, USA), anti-Actin (1:2,000, \#A5060, Sigma, St. Louis). Protein concentrations were measured by BCA Protein Assay Kit (CWBIO, Beijing, China). Protein samples were separated by SDS-PAGE and 
transferred to nitrocellulose membrane. After blocking with $5 \%$ skim milk, the membranes were incubated with first antibodies, followed by peroxidase conjugated secondary antibody. The signals were visualized with ECL reagent (Promega). The intensity of each Western blot band was analyzed by densitometric measurements (Image J, NIH). Data were normalized to GAPDH or Actin immunolabeling and expressed as percentage of control values.

\section{Immunohistochemistry}

KA injected and control mice were deeply anesthetized with pentobarbital, and their brains were rapidly removed, washed with PBS, incubated for at least $48 \mathrm{~h}$ in $4 \%$ paraformaldehyde (Sigma, Shanghai) and embedded with paraffin. Paraffinembedded tissues were sectioned at $4 \mu \mathrm{m}$ and mounted on precoated glass slides (GoldenBridge, Shanghai, China). The following primary antibodies were used: anti-Notch1 (1:100, \#SC-23299, Santa Cruz Biotechnology, Santa Cruz), antiJagged1 (1:200, \#SC-8303, Santa Cruz Biotechnology, Santa Cruz), anti-Tuj1 (1:200, \#ab7751, Abcam, Cambridge, MA, USA), and anti-Notch1 intracellular domain (1:200, \#1935-1, Epitomics, Burlingame). For immunofluorescence assays, after incubation with the mixed primary antibodies, the slices were incubated with secondary antibody conjugated with Alexa 488 and Alexa 594 (1:2,000, Molecular Probes, Eugene, OR, USA) to detect Notch1 and Tuj1, or Jagged1 and Tuj1. DAPI (Molecular Probes, Eugene) was used as a nuclear counterstain. All images were captured using a Leica inverted fluorescence microscope (DMI4000B, Leica, Wetzlar, Germany).

Hippocampal sections were immunostained and imaged with a confocal microscope (FV1000, Olympus). No threshold or other adjust was used as the soma is clearly delineated from background. For quantification in brain sections, areas were chosen randomly within the dentate gyrus which contains $50-60$ granule cells counted manually by DAPI. The areas usually have $400 \times 400$ pixels in control hippocampus and $500 \times 500$ pixels in the sclerotic hippocampus due to dentate gyrus dispersion. The average staining intensities were measured with ImageJ software. Data were acquired from 600-750 neurons of 12-15 fields from three experiments and presented as the average staining intensity of 50 granule cells. Student's $t$ test was used to determine the $p$ values.

\section{Hippocampal Slice Preparation and Electrophysiology}

We first prepared the acute model of seizures pretreated with Jagged 1 or DAPT, and the corresponding vehicles using C57BL6 mice (4-5 weeks old) as described above. Hippocampal slices $(300 \mu \mathrm{m})$ were prepared from mice 30 min after KA injection that were anesthetized by injecting pentobarbital $(50 \mathrm{mg} / \mathrm{kg})$ and decapitated with a guillotine.
The hippocampal slices were cut by a Vibratome in the modified and oxygenized artificial cerebrospinal fluid (ACSF) (mM: $124 \mathrm{NaCl}, 3 \mathrm{KCl}, 1.2 \mathrm{NaH} 2 \mathrm{PO} 4,26 \mathrm{NaHCO}, 0.5$ $\mathrm{CaCl} 2,5 \mathrm{MgSO} 4,20$ dextrose, and 5 HEPES; $\mathrm{pH}$ 7.35) at $4{ }^{\circ} \mathrm{C}$ and were held in the normal oxygenated ACSF (mM: $126 \mathrm{NaCl}, 2.5 \mathrm{KCl}, 1.2 \mathrm{NaH} 2 \mathrm{PO} 4,26 \mathrm{NaHCO} 3,2.0 \mathrm{CaCl}$, $2.0 \mathrm{MgSO} 4,10$ dextrose, and 5 HEPES; $\mathrm{pH} 7.35) 37^{\circ} \mathrm{C}$ for $1 \mathrm{~h}$ before the experiments. A slice was transferred to a submersion chamber (Warner RC-26G) that was perfused with normal ACSF for the electrophysiological experiments.

\section{Electrophysiological Studies in CA1 Pyramidal Neurons}

Electrophysiological studies were performed as previously described $[35,36]$. These pyramidal neurons were recorded by an amplifier (MultiClapm-700B, Axon Instrument Inc, Foster City, CA USA) under whole-cell current clamp and voltage clamp. Electrical signals were inputted into pClamp10 (Axon Instrument Inc., Foster City) with $100 \mathrm{kHz}$ of sampling rate. In our studies, transient capacitance was compensated, and output bandwidth was $3 \mathrm{kHz}$. The functions of pyramidal neurons were evaluated based on their sensitivity to excitatory inputs and their intrinsic properties (e.g., inputoutput spikes, thresholds). Pipette solutions for recording action potentials and excitatory post-synaptic currents included (mM) 150 K-gluconate, $5 \mathrm{NaCl}$, 0.4 EGTA, 4 Mg-ATP, 0.5 Tris-GTP, 4 Na-phosphocreatine, and 10 HEPES (pH 7.4 adjusted by $2 \mathrm{M} \mathrm{KOH}$ ). The pipette resistance was $8-10 \mathrm{M} \Omega$.

In the analyses of neuronal input-output, the depolarization pulses $(1 \mathrm{~s})$ in various stimulus intensities were injected into the neurons to induce action potential. By changing depolarization intensities, we measured input-output at each of pyramidal neurons in CA1. The sensitivity of pyramidal neurons in CA1 to excitatory inputs was evaluated by recording the activities of excitatory synapses on these neurons, in which sEPSC were recorded under voltage clamp. The isolation of sEPSC was done by applying $10 \mu \mathrm{M}$ bicuculline in perfusion solution. The cumulative probability of sEPSC amplitudes and inter-EPSC intervals was analyzed to estimate the responses of pyramidal neurons to excitatory inputs.

\section{Synaptic Vesicle Endocytosis Assay Using FM4-64}

FM4-64 is a membrane-selective fluorescent dye that has been widely used to observe synaptic vesicle recycling $[15,16]$. We used the dye to monitor synaptic endocytosis and to determine whether DAPT treatment could impair this process. FM4-64 is nonfluorescent in the aqueous phase and is intensely fluorescent when the dyes insert the lipophilic end into the cell membranes. During endocytosis following nerve electrical or chemical stimulation, the dyes become trapped inside the vesicle. Thus, after washing off the dyes on the cell surface membranes, the fluorescent signal is proportional to the 
number of newly formed vesicles $[37,38]$. Neuronal cultures were prepared from the hippocampus of E18 mouse embryos and plated on poly-L-lysine-coated $60-\mathrm{mm}$ dishes or $18-\mathrm{mm}$ glass coverslips. Neurons were exposed to pharmacological manipulations after 10 DIV. Neuronal cultures were treated with DAPT $(40 \mu \mathrm{M})$ or DMSO for $1 \mathrm{~h}$, and stimulated with KA $(500 \mu \mathrm{M})$ or saline for another $2 \mathrm{~h}$. Thereafter, FM4-64 stock (Biotium, Hayward, CA, USA) was added to the culture medium with a final concentration of $4 \mu \mathrm{M}$ and incubated for different intervals (30 and $120 \mathrm{~s}$ ). After incubation, the dyecontaining medium was discarded, and the neuronal culture was washed three times for 1 min each with PBS to remove surface-bound dye. Finally, the neuronal culture was incubated with Na-basic solution (Neurobasal medium containing $170 \mathrm{mM} \mathrm{NaCl} / 3.5 \mathrm{mM} \mathrm{KCl}$ ) for $60 \mathrm{~min}$ to repolarize the hippocampal neurons. This allows dye-loaded synaptic vesicles to complete recycling and minimize spontaneous synaptic activity. Fluorescent spots were visible within the nerve terminal with low background fluorescence. The spots represent clusters of synaptic vesicles containing a few hundred to several thousand vesicles. For each treatment culture, fluorescent images were obtained from at least eight randomly selected fields $(650 \mu \mathrm{m} \times 900 \mu \mathrm{m})$. ImageJ software (NIH) was used to quantify fluorescence intensity of FM4-64. The images were also applied for puncta quantifications. Acquired pictures were converted to 8 bits and auto-thresholded (see Fig. 6C), and the number of puncta were quantified using in-built Analyze Particles function of ImageJ software (pixel range was set from 4 to 40 to exclude noise). Data are from six to eight fields from three experiments. One-way ANOVA was used for statistical analysis. The data are presented as mean \pm SEM.

\section{Statistical Analysis}

All data are presented as the mean $\pm \operatorname{SEM}(n=$ number of individual samples). Statistic analysis was performed using Graph-Pad Software. The effects of treatments were analyzed using Student's $t$ test or Mann-Whitney test when comparing two groups (drug test and western blot of human hippocampi), and by one-way ANOVA followed by Dunnett's test when comparing more than two groups (western blot of mice hippocampi). Kolmogorov-Smirnov (KS) test was used to compare the cumulative probability curves for inter-EPSC intervals and amplitude between various treatment groups. Significance was accepted at $P<0.05$.

Acknowledgments We sincerely thank all the patients for their support and participation. We thank Prof. Jun Wei for revising of the manuscript. This work was supported by the National Basic Research Program of China [2010CB529603, 2012CB517902], National Natural Science
Foundation of China [30971001, 31222031, 31021091], Beijing Natural Science Foundation [7102109], and Fok Ying Tong Education Foundation [121024].

Conflict of Interest The authors declare that they have no conflict of interest.

\section{References}

1. Ables JL, Breunig JJ, Eisch AJ, Rakic P (2011) Not(ch) just development: Notch signalling in the adult brain. Nat Rev Neurosci 12(5): 269-283. doi:10.1038/nrn3024

2. Pierfelice T, Alberi L, Gaiano N (2011) Notch in the vertebrate nervous system: an old dog with new tricks. Neuron 69(5):840 855. doi:10.1016/j.neuron.2011.02.031

3. Alberi L, Liu S, Wang Y, Badie R, Smith-Hicks C, Wu J, Pierfelice TJ, Abazyan B, Mattson MP, Kuhl D, Pletnikov M, Worley PF, Gaiano N (2011) Activity-induced Notch signaling in neurons requires Arc/ Arg3.1 and is essential for synaptic plasticity in hippocampal networks. Neuron 69(3):437-444. doi:10.1016/j.neuron.2011.01.004

4. Lieber T, Kidd S, Struhl G (2011) DSL-Notch signaling in the Drosophila brain in response to olfactory stimulation. Neuron 69(3):468-481. doi:10.1016/j.neuron.2010.12.015

5. Hortopan GA, Baraban SC (2011) Aberrant expression of genes necessary for neuronal development and Notch signaling in an epileptic mind bomb zebrafish. Dev Dyn: Off Publ Am Assoc Anat 240(8):1964-1976. doi:10.1002/dvdy.22680

6. Jiang YH, Armstrong D, Albrecht U, Atkins CM, Noebels JL, Eichele G, Sweatt JD, Beaudet AL (1998) Mutation of the Angelman ubiquitin ligase in mice causes increased cytoplasmic p53 and deficits of contextual learning and long-term potentiation. Neuron 21(4): 799-811

7. Hortopan GA, Dinday MT, Baraban SC (2010) Spontaneous seizures and altered gene expression in GABA signaling pathways in a mind bomb mutant zebrafish. J Neurosci Off J Soc Neurosci 30(41): 13718-13728. doi:10.1523/JNEUROSCI.1887-10.2010

8. Riban V, Bouilleret V, Pham-Le BT, Fritschy JM, Marescaux C, Depaulis A (2002) Evolution of hippocampal epileptic activity during the development of hippocampal sclerosis in a mouse model of temporal lobe epilepsy. Neuroscience 112(1):101-111

9. Bouilleret V, Ridoux V, Depaulis A, Marescaux C, Nehlig A, Le Gal La Salle G (1999) Recurrent seizures and hippocampal sclerosis following intrahippocampal kainate injection in adult mice: electroencephalography, histopathology and synaptic reorganization similar to mesial temporal lobe epilepsy. Neuroscience 89(3):717-729

10. Breunig JJ, Silbereis J, Vaccarino FM, Sestan N, Rakic P (2007) Notch regulates cell fate and dendrite morphology of newborn neurons in the post-natal dentate gyrus. Proc Natl Acad Sci U S A 104(51):20558-20563. doi:10.1073/pnas.0710156104

11. Balosso S, Ravizza T, Perego C, Peschon J, Campbell IL, De Simoni MG, Vezzani A (2005) Tumor necrosis factor-alpha inhibits seizures in mice via p75 receptors. Ann Neurol 57(6):804-812. doi:10.1002/ ana.20480

12. Balosso S, Maroso M, Sanchez-Alavez M, Ravizza T, Frasca A, Bartfai T, Vezzani A (2008) A novel non-transcriptional pathway mediates the proconvulsive effects of interleukin-1beta. Brain J Neurol 131(Pt 12):3256-3265. doi:10.1093/brain/awn271

13. Warnock DE, Schmid SL (1996) Dynamin GTPase, a forcegenerating molecular switch. BioEssays 18(11):885-893. doi:10. 1002/bies.950181107

14. Sudhof TC (2004) The synaptic vesicle cycle. Annu Rev Neurosci 27:509-547. doi:10.1146/annurev.neuro.26.041002.131412 
15. Meyers JR, MacDonald RB, Duggan A, Lenzi D, Standaert DG, Corwin JT, Corey DP (2003) Lighting up the senses: FM1-43 loading of sensory cells through nonselective ion channels. J Neurosci Off $\mathbf{J}$ Soc Neurosci 23(10):4054-4065

16. Bolte S, Talbot C, Boutte Y, Catrice O, Read ND, Satiat-Jeunemaitre B (2004) FM-dyes as experimental probes for dissecting vesicle trafficking in living plant cells. J Microsc 214(Pt 2):159-173. doi: 10.1111/j.0022-2720.2004.01348.x

17. Salama-Cohen P, Arevalo MA, Grantyn R, Rodriguez-Tebar A (2006) Notch and NGF/p75NTR control dendrite morphology and the balance of excitatory/inhibitory synaptic input to hippocampal neurones through Neurogenin 3. J Neurochem 97(5):1269-1278. doi:10.1111/j.1471-4159.2006.03783.x

18. Wang Y, Chan SL, Miele L, Yao PJ, Mackes J, Ingram DK, Mattson MP, Furukawa K (2004) Involvement of Notch signaling in hippocampal synaptic plasticity. Proc Natl Acad Sci U S A 101(25):9458-9462. doi:10.1073/pnas.0308126101

19. Hinshaw JE (2000) Dynamin and its role in membrane fission. Annu Rev Cell Dev Biol 16:483-519. doi:10.1146/annurev.cellbio.16.1.483

20. Xu B, Li S, Brown A, Gerlai R, Fahnestock M, Racine RJ (2003) EphA/ ephrin-A interactions regulate epileptogenesis and activity-dependent axonal sprouting in adult rats. Mol Cell Neurosci 24(4):984-999

21. Matsui C, Inoue E, Kakita A, Arita K, Deguchi-Tawarada M, Togawa A, Yamada A, Takai Y, Takahashi H (2012) Involvement of the gamma-secretase-mediated EphA4 signaling pathway in synaptic pathogenesis of Alzheimer's disease. Brain Pathol 22(6):776-787. doi:10.1111/j.1750-3639.2012.00587.x

22. Wei J, Hemmings GP (2000) The NOTCH4 locus is associated with susceptibility to schizophrenia. Nat Genet 25(4):376-377. doi:10. $1038 / 78044$

23. Chang YT, Chen PC, Tsai IJ, Sung FC, Chin ZN, Kuo HT, Tsai CH, Chou IC (2011) Bidirectional relation between schizophrenia and epilepsy: a population-based retrospective cohort study. Epilepsia 52(11):2036-2042. doi:10.1111/j.1528-1167.2011.03268.x

24. Yizhar O, Fenno LE, Prigge M, Schneider F, Davidson TJ, O'Shea DJ, Sohal VS, Goshen I, Finkelstein J, Paz JT, Stehfest K, Fudim R, Ramakrishnan C, Huguenard JR, Hegemann P, Deisseroth K (2011) Neocortical excitation/inhibition balance in information processing and social dysfunction. Nature 477(7363):171-178. doi:10.1038/ nature 10360

25. Dudek FE (2009) Epileptogenesis: a new twist on the balance of excitation and inhibition. Epilepsy Curr Am Epilepsy Soc 9(6):174 176. doi:10.1111/j.1535-7511.2009.01334.x

26. Toro CT, Deakin JF (2007) Adult neurogenesis and schizophrenia: a window on abnormal early brain development? Schizophr Res 90(13):1-14. doi:10.1016/j.schres.2006.09.030
27. Kuruba R, Hattiangady B, Shetty AK (2009) Hippocampal neurogenesis and neural stem cells in temporal lobe epilepsy. Epilepsy Behav E\&B 14(Suppl 1):65-73. doi:10.1016/j.yebeh.2008.08. 020

28. Ledergerber D, Fritschy JM, Kralic JE (2006) Impairment of dentate gyrus neuronal progenitor cell differentiation in a mouse model of temporal lobe epilepsy. Exp Neurol 199(1):130-142. doi:10.1016/j. expneurol.2006.02.010

29. Kralic JE, Ledergerber DA, Fritschy JM (2005) Disruption of the neurogenic potential of the dentate gyrus in a mouse model of temporal lobe epilepsy with focal seizures. Eur J Neurosci 22(8): 1916-1927. doi:10.1111/j.1460-9568.2005.04386.x

30. Elliott RC, Khademi S, Pleasure SJ, Parent JM, Lowenstein DH (2001) Differential regulation of basic helix-loop-helix mRNAs in the dentate gyrus following status epilepticus. Neuroscience 106(1): $79-88$

31. Sibbe M, Haussler U, Dieni S, Althof D, Haas CA, Frotscher M (2012) Experimental epilepsy affects Notch1 signalling and the stem cell pool in the dentate gyrus. Eur J Neurosci 36(12):3643-3652. doi: 10.1111/j.1460-9568.2012.08279.x

32. Parent JM, Elliott RC, Pleasure SJ, Barbaro NM, Lowenstein DH (2006) Aberrant seizure-induced neurogenesis in experimental temporal lobe epilepsy. Ann Neurol 59(1):81-91. doi: 10.1002/ana.20699

33. Rensing NR, Guo D, Wong M (2012) Video-EEG monitoring methods for characterizing rodent models of tuberous sclerosis and epilepsy. Methods Mol Biol 821:373-391. doi:10.1007/978-161779-430-8 24

34. Maroso M, Balosso S, Ravizza T, Liu J, Aronica E, Iyer AM, Rossetti C, Molteni M, Casalgrandi M, Manfredi AA, Bianchi ME, Vezzani A (2010) Toll-like receptor 4 and high-mobility group box-1 are involved in ictogenesis and can be targeted to reduce seizures. Nat Med 16(4):413-419. doi:10.1038/nm.2127

35. Ge R, Qian H, Wang JH (2011) Physiological synaptic signals initiate sequential spikes at soma of cortical pyramidal neurons. Mol Brain 4: 19. doi:10.1186/1756-6606-4-19

36. Wang JH, Zhang MJ (2004) Differential modulation of glutamatergic and cholinergic synapses by calcineurin in hippocampal CA1 fastspiking interneurons. Brain Res 1004(1-2):125-135. doi:10.1016/j. brainres.2004.01.025

37. Zakharenko SS, Zablow L, Siegelbaum SA (2001) Visualization of changes in presynaptic function during long-term synaptic plasticity. Nat Neurosci 4(7):711-717. doi:10.1038/89498

38. Anggono V, Cousin MA, Robinson PJ (2008) Styryl dye-based synaptic vesicle recycling assay in cultured cerebellar granule neurons. Methods Mol Biol 457:333-345 